\title{
Détermination de l'activité acidifiante des suspensions concentrées congelées de bactéries lactiques ${ }^{*}$
}

\author{
par \\ J.P. ACCOLAS et J. AUCLAIR \\ Station Centrale de Recherches Laitières et de Technologie \\ des Produits Animaux, I.N.R.A. (78) Jouy-en-Josas
}

\section{I. - INTRODUCTION}

Depuis quelques années, un certain nombre de travaux poursuivis en France et à l'étranger ont été consacrés à la préparation de suspensions concentrées de bactéries lactiques, suspensions desti- . nées à simplifier ou même à supprimer la préparation traditionnelle des levains sur lait telle qu'elle est pratiquée actuellement dans l'industrie laitière $[1,5,10,12,13,15,16,17,18]$. Ces suspensions bactériennes concentrées sont conservées soit par congélation à des températures moyennement basses $\left(-20^{\circ} \mathrm{C}\right.$ à $\left.-40^{\circ} \mathrm{C}\right)[1,10,16,17]$, soit par congélation dans l'azote liquide [12], soit à l'état sec par lyophilisation ou simple séchage $[11,16]$.

Deux sortes de déterminations permettent d'apprécier la « qualité » des suspensions bactériennes concentrées utilisées : d'une part la mesure du nombre de germes viables qu'elles contiennent (on parle dans ce cas de la viabilité des suspensions) ; d'autre part leur aptitude à produire rapidement de l'acide lactique à partir du lactose du lait ; c'est ce que l'on appelle généralement leur activité acidifiante.

Cette activité acidifiante dépend à la fois du nombre de cellules bactériennes présentes dans l'unité de volume de la suspension et de l'état physiologique de ces cellules, ce dernier étant lui-même fonction de nombreux facteurs liés à l'espèce ou même à la souche bactérienne, aux conditions de culture, au stade de la croissance, aux traitements éventuels subis par les cellules, comme l'ont montré en particulier les travaux de Bergère $[4,6]$ et de Bergère et Hermier [5] sur les streptocoques lactiques mésophiles.

* Ce travail a bénéficié d'une subvention de la Délégation Générale à la Recherche Scientifique et Technique (Contrat 67-00-998). 
La détermination de l'activité des suspensions de bactéries lactiques est relativement délicate et son interprétation souvent difficile. En fait, si l'on considère les différentes publications récentes relatives à la mesure de l'activité des suspensions concentrées ou des cultures de bactéries lactiques, on constate de grandes variations dans les méthodes utilisées aussi bien que dans l'interprétation des résultats qui en est faite.

Certains auteurs $[10,11,12]$ ont, par exemple, comparé les deux courbes d'acidification obtenues l'une à partir d'un inoculum provenant d'une culture sur lait, l'autre à partir d'un inoculum comparable, après dilution éventuelle, de la suspension bactérienne de la même souche. Cette méthode de comparaison permet d'avoir une idée approximative de l'activité de la suspension étudiée, par rapport à l'activité de la culture témoin. Son caractère semi-quantitatif, souvent suffisant en pratique, restreint toutefois les comparaisons à des limites étroites.

Une expression quantitative de l'activité acidifiante est souvent utilisée dans de nombreux travaux $[2,3,7,8,14,16]$. Pour comparer l'activité de deux suspensions de bactéries lactiques, on exprime le rapport existant entre les quantités d'acide produites par chacune des suspensions, dans des conditions déterminées (inoculum, temps et température d'incubation), la production d'acide de l'une des suspensions étant arbitrairement fixée à 100 .

Ce mode d'expression implique que l'activité des suspensions est proportionnelle à la quantité d'acide formée en un temps donné par un inoculum déterminé. En fait il n'est applicable que dans des limites très étroites, pour la comparaison de suspensions dont l'activité est voisine. Il suppose en outre que, pour toutes les espèces (et souches) de bactéries lactiques, la production d'acide obéit à la même relation, ce qui n'est évidemment pas le cas.

Une expression quantitative plus satisfaisante de l'activité acidifiante des bactéries lactiques a fait l'objet de mises au point récentes. En particulier Bergère [4] a décrit une méthode de détermination de l'activité des streptocoques lactiques mésophiles, méthode que nous avons nous-mêmes utilisée dans un précédent travail [1]. De même Gavin [9] a décrit une méthode analogue dans son principe, pour déterminer l'activité acidifiante de Lactobacillus bulgaricus et a utilisé, sans la décrire, une méthode équivalente pour Streptococcus thermophilus. Ces modes d'expression reposent sur le rapport existant entre les quantités de deux suspensions $A_{1}$ et $A_{2}$ d'une même souche de bactéries lactiques donnant, dans les mêmes conditions, une production d'acide identique. Cela revient à dire, par exemple, que la suspension $A_{1}$ est deux fois (ou dix fois) plus active que la suspension $A_{2}$ si la suspension $A_{1}$ doit être diluée deux fois (ou dix fois) plus que la suspension $\mathrm{A}_{2}$ pour obtenir la même production d'acide. 
Ce mode d'expression est, nous semble-t-il, le plus logique. C'est d'ailleurs sur ce même principe que sont basées la plupart des méthodes de mesure de l'activité des substances stimulantes (telles que les vitamines), de l'activité des substances inhibitrices (par exemple les antibiotiques) ou encore de l'activité des préparations enzymatiques.

Nous avons donc cherché, dans le présent travail, à mettre au point une méthode de mesure de l'activité acidifiante des bactéries lactiques basée sur le principe que nous venons de mentionner et répondant par ailleurs à un double objectif : En premier lieu cette méthode devrait se caractériser par une simplicité de mise en œuvre évitant de recourir à un matériel complexe et coûteux ; cela nous a orienté vers la mesure de l'acidité titrable plutôt que vers la mesure du $\mathrm{pH}$. En second lieu la reproductibilité et la précision de cette méthode devaient être évaluées, pour permettre d'en fixer les limites d'interprétation.

Nous avons considéré ici comme bactéries lactiques aussi bien les bactéries mésophiles des espèces Streptococcus lactis, Str. cremoris, Str. diacetilactis, que les lactobacilles thermophiles (Lactobacillus helveticus, L. lactis, L. bulgaricus) et également Str. thermophilus.

Les suspensions concentrées qui ont fait l'objet de cette étude étaient préparées selon des techniques analogues à celles décrites précédemment [1, 17], à partir de souches de bactéries lactiques appartenant à la collection du Centre National de Recherches Zootechniques.

\section{II. - PRINCIPE DE LA MESURE D'ACTIVITE}

La détermination de l'activité repose sur la mesure de la quantité d'acide lactique produite, en un temps déterminé, dans des cultures sur lait, inoculées avec des dilutions successives de la suspension. On utilise une incubation de $6 \mathrm{~h}$ à $30^{\circ} \mathrm{C}$ pour les streptocoques mésophiles et une incubation de $4 \mathrm{~h} 30$ à $42^{\circ} \mathrm{C}$ pour les streptocoques et les lactobacilles thermophiles.

Pour une souche déterminée de bactéries lactiques, les résultats expérimentaux montrent que :

1) Il existe une relation linéaire entre la quantité d'acide produite et le facteur de dilution, tout au moins dans certaines limites de dilutions qui sont variables avec les souches (fig. 1, courbe A). Autrement dit, la production d'acide d'une suspension de bactéries lactique est, dans certaines limites, proportionnelle au logarithme de la quantité de bactéries initialement présentes dans l'inoculum.

2) Si l'on examine, dans les mêmes conditions, deux suspensions différentes A et B d'une même souche de bactéries lactiques, on 
obtient par représentation graphique, des portions de droites parallèles (fig. 1). La distance séparant ces deux droites permet d'exprimer quantitativement le rapport existant entre les activités de A et $\mathrm{B}$. Dans le cas où les dilutions utilisées suivent une progression géométrique de raison $1 / 2$, l'expression mathématique du rapport $r$

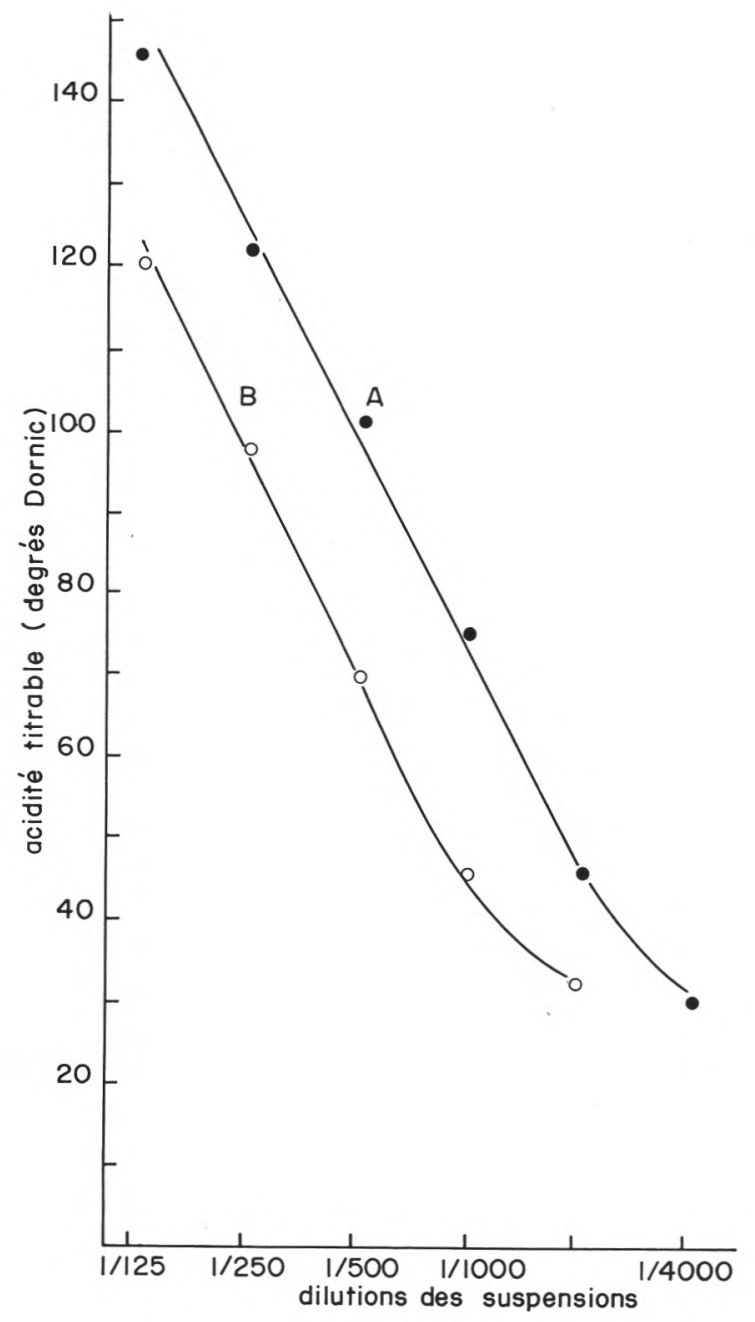

fig. 1

Variations de la quantité d'acide produite par deux suspensions A et B de $L$. helveticus 303 , en fonction de l'inoculum. 
existant entre les activités de A et de B peut être formulée de la façon suivante* :

$$
\mathrm{r}=\frac{\text { activité de } \mathrm{A}}{\text { activité de } \mathrm{B}}=10 \frac{\mathrm{a}_{1}-\mathrm{b}_{1}}{\mathrm{a}_{1}-\mathrm{a}_{2}} \times 0,301
$$

où $\mathrm{a}_{1}=$ quantité d'acide produite par la suspension $\mathrm{A}$ à la dilution $1 / \mathrm{n}$.

$\mathrm{a}_{2}=$ quantité d'acide produite par la suspension $\mathrm{A}$ à la dilution $1 / 2 \mathrm{n}$.

$\mathrm{b}_{1}=$ quantité d'acide produite par la suspension $\mathrm{B}$ à la dilution $1 / \mathrm{n}$.

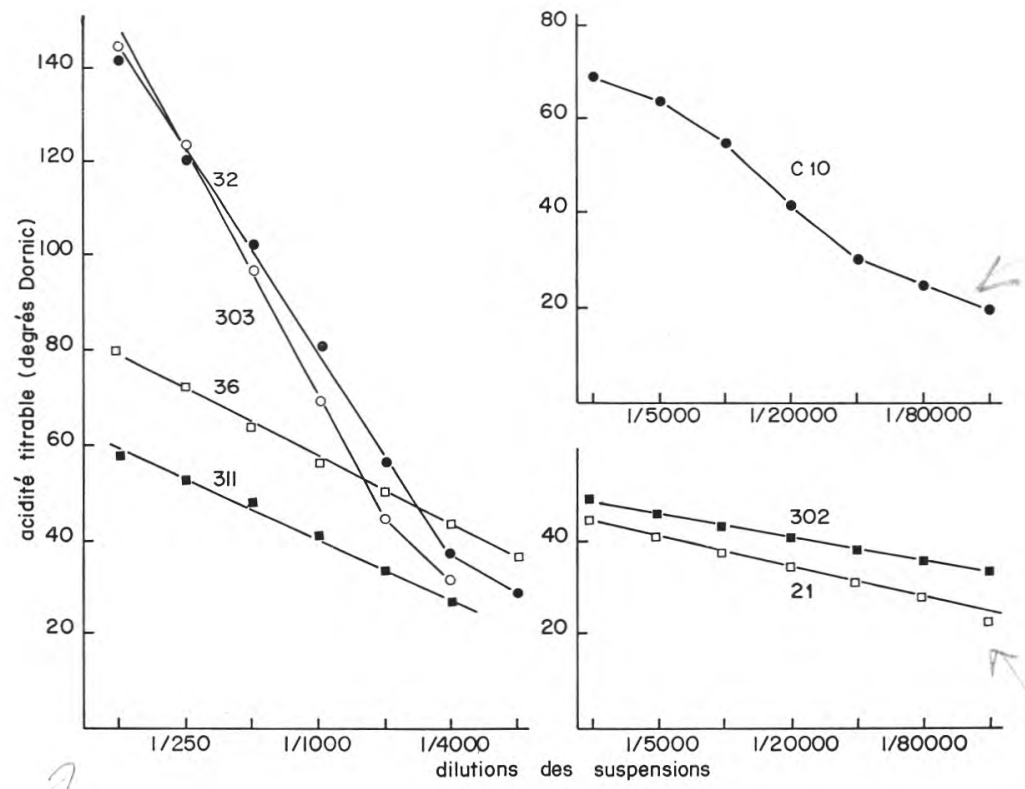

fig. 2

Variations de la quantité d'acide produite par différentes suspensions de bactéries lactiques, en fonction de l'inoculum.

L. bulgaricus 36, L. lactis 311, L. helveticus 32 et 303.

Str. thermophilus 21 et 302 .

Str. lactis C 10 .

La figure 2 montre, à titre d'exemple, les résultats obtenus avec des suspensions de Str. lactis, de Str. thermophilus et de différentes espèces de lactobacilles.

* Voir en annexe la formulation détaillée de l'expression mathématique de l'activité et la description du mode de détermination graphique. 
Pour comparer plus aisément entre elles plusieurs suspensions d'une même souche bactérienne, on choisit une suspension de référence, dont l'activité est fixée arbitrairement à 100 unités par g. Cette suspension est caractérisée par sa droite de référence.

Les droites de référence correspondant aux différentes souches étudiées ont été définies par les paramètres suivants :

- Str. lactis C 10

Production d'acide à la dilution $1 / 10000 \ldots \ldots \ldots \ldots$

Différence entre les quantités d'acide produites par deux dilutions successives de $1 / 2$ en $1 / 2 \ldots \ldots \ldots \ldots \ldots \ldots \ldots \ldots 2^{\circ} \mathrm{D}$

- Str. thermophilus 21 et 302

Production d'acide à la dilution $1 / 10000 \ldots \ldots \ldots \ldots 40^{\circ} \mathrm{D}$

Différence entre les quantités d'acide produites par deux dilutions successives de $1 / 2$ en $1 / 2$ :

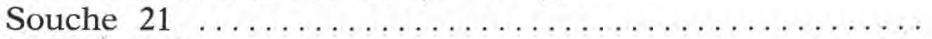

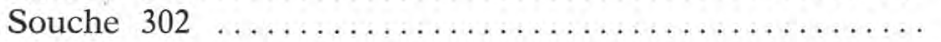

- L. helveticus 32 et 303

Production d'acide à la dilution $1 / 1000$

Différence entre les quantités d'acide produites par deux dilutions successives de $1 / 2$ en $1 / 2$ :

Souche 32

Souche 303

- L. bulgaricus 36

Production d'acide à la dilution $1 / 1000 \ldots \ldots \ldots \ldots 60^{\circ} \mathrm{D}$

Différence entre les quantités d'acide produites par deux dilutions successives de $1 / 2$ en $1 / 2 \ldots \ldots \ldots \ldots \ldots \ldots, 7,5^{\circ} \mathrm{D}$

- L. lactis 311

Production d'acide à la dilution 1/1000

Différence entre les quantités d'acide produites par deux dilutions successives de $1 / 2$ en $1 / 2 \ldots \ldots \ldots \ldots \ldots \ldots$

Pour exprimer en unités l'activité de différentes suspensions d'une souche donnée on peut utiliser une représentation graphique en coordonnées semi-logarithmiques telles qu'elle est donnée sur la figure 3 à titre d'exemple pour L. helveticus 303 .

\section{III. - MODE OPERATOIRE}

\section{Lait}

On utilise du lait écrémé en poudre à solubilité instantanée, exempt d'antibiotique. Pour limiter les variations imputables à la qualité du lait, nous avons utilisé un même lot de poudre de lait conservé au réfrigérateur en boîtes métalliques, pendant une période de l'ordre de deux ans. Dans ces conditions, nous n'avons pas observé de variations notables. 
Le lait est reconstitué à raison de $100 \mathrm{~g}$ de poudre pour $1000 \mathrm{ml}$ d'eau distillée. Le lait reconstitué est réparti en flacons de $125 \mathrm{ml}$ bouchés à vis, à raison de $100 \mathrm{ml}$ par flacon. Dans le cas des streptocoques lactiques mésophiles, les flacons de lait sont chauffés dans un bain-marie réglé à $80^{\circ} \mathrm{C}$, penđant $30 \mathrm{mn}$. Dans le cas des bactéries lactiques thermophiles, streptocoques et lactobacilles, les flacons de lait sont autoclavés à $110^{\circ} \mathrm{C}$ pendant $10 \mathrm{mn}$.

Après le traitement thermique, les flacons de lait sont refroidis rapidement et conservés au réfrigérateur jusqu'à l'utilisation, au plus tard le lendemain de la préparation.

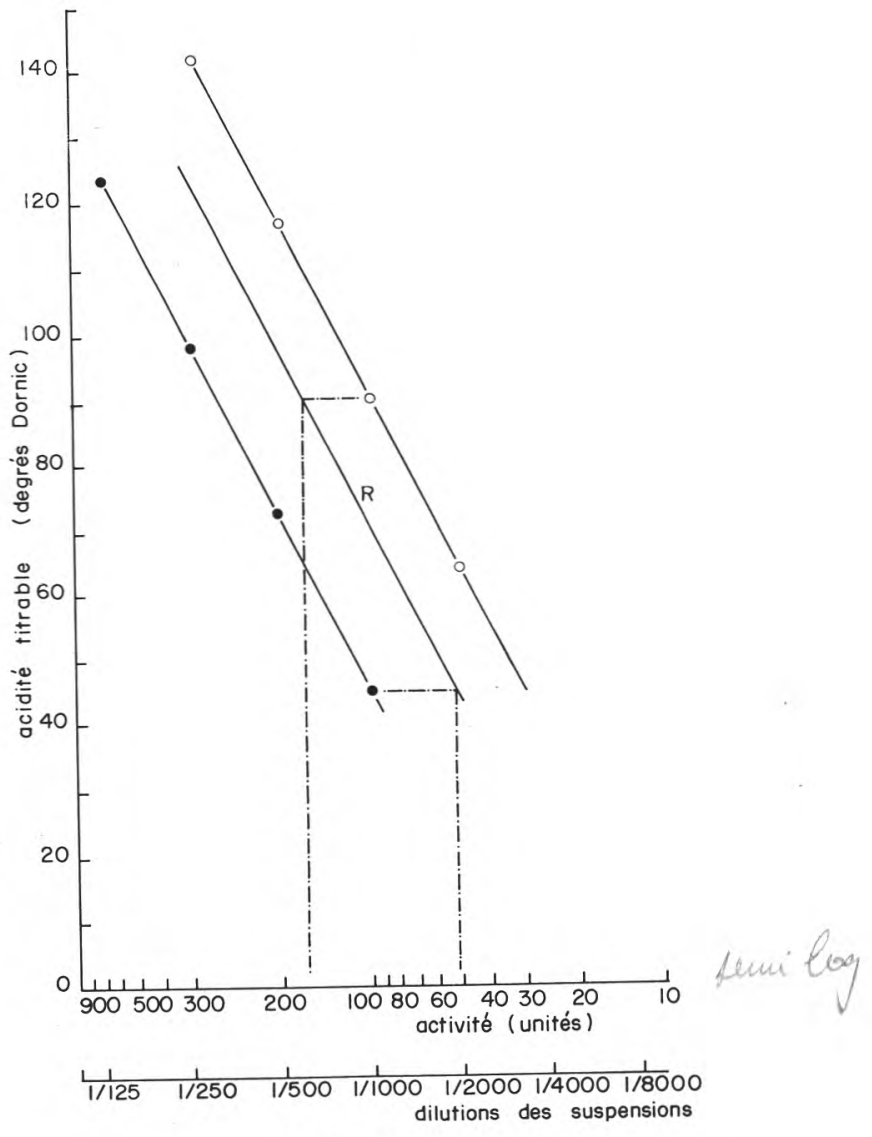

fig. 3

Détermination de l'activité des suspensions de L. helveticus 303 $\mathrm{R}$ : Suspension de référence.

$\bigcirc-\bigcirc$ et : Suspensions inconnues. La détermination graphique donne des titres de 170 et 51 unités respectivement. 


\section{Dilutions}

L'examen portant généralement sur des suspensions bactériennes conservées à l'état congelé, ces suspensions sont au préalable décongelées rapidement dans un bain-marie à une température comprise entre 40 et $42^{\circ} \mathrm{C}$, en agitant constamment.

Le lait utilisé pour les dilutions ainsi que les tubes ensemencés sont maintenus dans l'eau glacée pendant toute la durée des manipulations précédant l'incubation. La première dilution, au 1/10 ou au $1 / 12,5$ suivant les cas, est effectuée en pesant sur un trébuchet de précision une quantité déterminée de suspension et la quantité correspondante de lait (par exemple $3 \mathrm{~g}$ de suspension et $27 \mathrm{~g}$ de lait pour une dilution au 1/10). A partir de cette première dilution, les dilutions successives suivant généralement une progression géométrique de raison $1 / 2$ sont effectuées selon une gamme, variable avec les souches utilisées. Le contenu de chaque tube de dilution est soigneusement agité à la pipette, en évitant la formation de mousse.

Les dilutions finales sont faites dans des tubes bouchés à vis de telle façon que les tubes contiennent, après inoculation, $15 \mathrm{ml}$ de lait. Les tubes de lait ensemencés sont agités une seule fois, par simple retournement du tube, avant incubation.

\section{Incubation}

Les tubes de lait ensemencés sont soigneusement immergés dans un bain-marie réglé à $\pm 0,1^{\circ} \mathrm{C}$. Pour les streptocoques lactiques mésophiles on utilise une incubation de $6 \mathrm{~h}$ à $30^{\circ} \mathrm{C}$. Pour les bactéries lactiques thermophiles, on utilise une incubation de 4 h 30 à $42^{\circ} \mathrm{C}$.

$\mathrm{Au}$ terme de la période d'incubation les tubes sont refroidis immédiatement dans l'eau glacée et maintenus à $0^{\circ} \mathrm{C}$ jusqu'au titrage.

\section{Titrage}

La mesure de l'acidité titrable est effectuée sur $9 \mathrm{~g}$ de chacune des cultures obtenues, pesés à l'aide d'un trébuchet de précision. On ajoute $0,5 \mathrm{ml}$ d'une solution à $1 \mathrm{p} .100(\mathrm{p} / \mathrm{v})$ de phénolphtaléine dans l'alcool à $95^{\circ}$. La titration est effectuée à l'aide de soude décinormale. L'acidité est exprimée en degrés Dornic. Dans les conditions du titrage $1 \mathrm{ml}$ de soude $0,1 \mathrm{~N}$ correspond à $10^{\circ} \mathrm{D}(1 \mathrm{~g}$ d'acide lactique par litre de lait).

\section{Expression des résultats}

On obtient de cette façon une série de valeurs, exprimées en degrés Dornic, correspondant à la production d'acide à partir d'une série d'inoculums différents de la suspension bactérienne. 
Sur un papier semi-logarithmique à deux modules, on représente les valeurs d'acidité ainsi obtenues (échelle arithmétique) en fonction de l'inoculum utilisé (échelle logarithmique). Si les dilutions ont été convenablement choisies, au moins trois des points obtenus doivent être alignés.

Cela permet :

1) de vérifier que la suspension examinée donne bien une droite parallèle à la droite de référence de la souche correspondante,

2) de calculer graphiquement, comme il est indiqué en annexe, le nombre d'unités d'activité contenues dans la suspension titrée.

\section{Remarque}

Pour une suspension connue, qui a déjà été titrée une ou plusieurs fois, on peut opérer plus simplement. C'est par exemple le cas d'une suspension dont on détermine régulièrement l'activité, au cours de la conservation à l'état congelé.

Dans ce cas, on peut n'utiliser qu'une seule dilution à condition que la quantité d'acide produite correspondant à cette dilution se situe dans la portion linéaire de la courbe. On peut effectuer l'opération de dilution en triplicata et utiliser la moyenne des trois valeurs obtenues. Le calcul du nombre d'unités d'activité s'effectue de la même manière que précédemment.

\section{IV. - REPRODUCTIBILITE ET PRECISION DE LA METHODE}

La reproductibilité et la précision des résultats expérimentaux obtenus varient avec les organismes étudiés (tab. 1). Ces différences sont liées à la fois à l'erreur expérimentale et à la pente de la droite de référence caractéristique de chaque espèce, voire de chaque souche de bactéries lactiques. Ainsi il est possible d'obtenir dans le cas de Str. thermophilus 21 et 302 des séries de valeurs dont la moyenne se situe dans un intervalle inférieur ou égal à $2^{\circ} \mathrm{D}$, mais, compte tenu de la faible pente de la droite de référence $\left(2,5^{\circ} \mathrm{D}\right.$ à $3^{\circ} \mathrm{D}$ pour deux dilutions successives de $1 / 2$ en 1/2) l'expression quantitative de l'activité se caractérisera par un coefficient de variation élevé (40 à 50 p. 100). Inversement, dans le cas de L. helveticus 32 et 303, la moyenne des résultats se situe dans un intervalle de $8^{\circ} \mathrm{D}$ mais, la pente de la droite de référence étant forte $\left(21\right.$ à $26^{\circ} \mathrm{D}$ pour deux dilutions successives de $1 / 2$ en $1 / 2$ ), le coefficient de variation se situera entre 10 et 15 p. 100 et la précision de la mesure sera meilleure. L. bulgaricus et les streptocoques lactiques mésophiles appartiennent à un groupe intermédiaire pour lequel le coefficient de variation se situe entre 10 et 30 p. 100 . 
TABLEAU 1. - Activité moyenne de différentes suspensions de bactéries lactiques

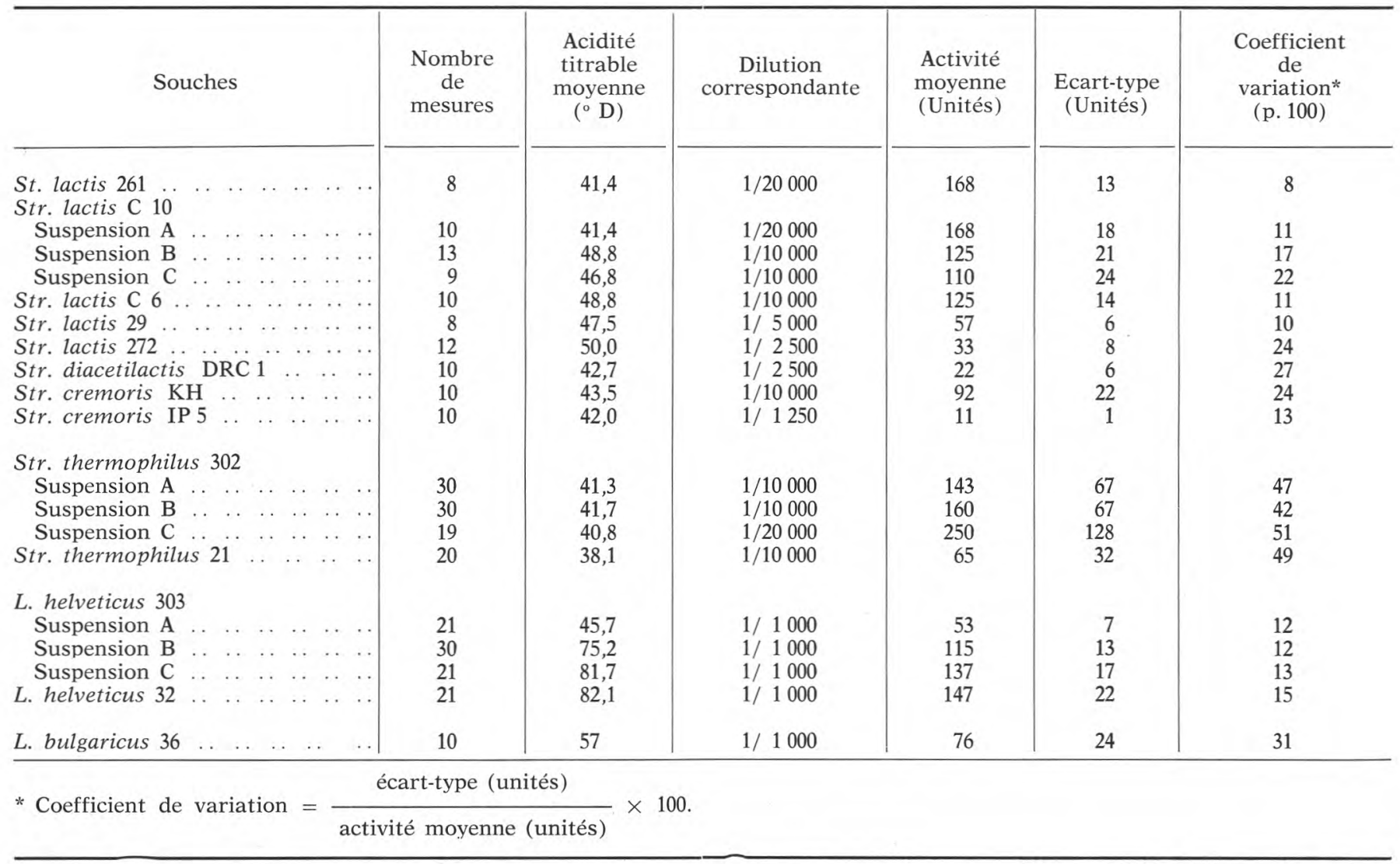


Il est bon de souligner que la reproductibilité de la méthode dépend étroitement des conditions expérimentales. En premier lieu il est nécessaire de pratiquer une homogénéisation soignée de chaque dilution, en particulier de la première dilution, homogénéisation qui peut être plus ou moins aisée suivant les suspensions étudiées.

En second lieu, il est nécessaire de maintenir le potentiel d'oxydoréduction initial des cultures sur lait à une valeur aussi constante que possible. Comme le montre, à titre d'exemple, le tableau 2 pour $L$. helveticus une aération plus ou moins poussée du lait, avant ou pendant la culture, peut provoquer des variations considérables de l'acidification, variations conduisant à des estimations totalement erronées de l'activité.

En troisième lieu, de petites variations du degré de chauffage du lait, dans le cas du lait autoclavé utilisé pour les bactéries lactiques thermophiles peuvent également être à l'origine de variations de l'acidification. L'effet stimulant bien connu du lait autoclavé sur des

\section{TABLEAU 2}

Variations de l'activité d'une suspension de $L$. helveticus avec le degré d'oxygénation du lait

\begin{tabular}{l|c|c|c}
\hline & $\begin{array}{c}\text { Acidité titrable } \\
(\circ \text { D) }\end{array}$ & $\begin{array}{c}\text { Activité* } \\
\text { (Unités) }\end{array}$ & $\begin{array}{c}\text { Variation } \\
(\mathrm{p} .100)\end{array}$ \\
\hline Lait A & 90 & 170 & - \\
Lait B & 88 & 160 & 6 \\
Lait C & 83 & 145 & 33 \\
Lait D & 75 & 114 & 60 \\
Lait E & 55 & 67 & \\
\hline
\end{tabular}

* Suspension de référence : 100 unités, $70^{\circ} \mathrm{D}$ au $1 / 1000$.

** Activité de l'essai A prise pour base 100 .

A partir d'un même lot de lait autoclavé la veille de l'essai $\left(110^{\circ} \mathrm{C}-10 \mathrm{mn}\right)$ en fioles de $100 \mathrm{ml}$ bouchées à vis, les différents échantillons ont été traités de la façon suivante :

A : Echantillon régénéré au bain-marie bouillant $15 \mathrm{mn}$, avant ensemencement.

B : Echantillon ensemencé sans traitement préalable.

C : Echantillon fortement aéré pendant $30 \mathrm{mn}$ sur un agitateur magnétique, puis régénéré au bain-marie bouillant $15 \mathrm{mn}$, avant ensemencement.

D : Echantillon fortement aéré comme l'échantillon $\mathrm{C}$, mais non régénéré avant ensemencement.

E : Echantillon fortement aéré comme les échantillons $C$ et $D$ avant ensemencement. De plus au cours de l'incubation les tubes sont fortement agités pendant $5 \mathrm{~s}$ toutes les $30 \mathrm{mn}$.

Inoculum : 1 p. 1000 d'une suspension de L. helveticus 303. 
bactéries lactiques telles que L. bulgaricus, L. lactis, Str. thermophilus, peut être plus ou moins marqué suivant que le lait a été plus ou moins chauffé. Les variations résultantes de l'acidification, même si elles ne sont pas excessives en valeur absolue ( 3 à $4^{\circ}$ D) limitent notablement la précision de la méthode dans le cas de ces microorganismes.

On peut en pratique se prémunir contre, ou au moins limiter l'effet de ces variations aléatoires en utilisant des "suspensions étalons » de bactéries lactiques, dont l'activité acidifiante est suffisamment stable. C'est le cas des suspensions conservées à l'état congelé dans l'azote liquide, dont l'activité se maintient sans variation notable pendant de très longues périodes de temps, un an ou probablement plusieurs années. En mesurant comparativement l'activité de telles suspensions, parallèlement à chaque série d'épreuves d'activité, il est possible de limiter les erreurs d'interprétation imputables aux variations aléatoires des conditions expérimentales de travail.

\section{V. - DISCUSSION}

La méthode que nous venons de décrire repose sur la relation linéaire existant entre la production d'acide d'une suspension en un temps donné et la dilution de cette suspension.

Il n'est cependant pas impossible que la portion de courbe que nous avons assimilée à une droite soit en réalité une portion de courbe présentant un point d'inflexion, en particulier dans le cas des streptocoques lactiques mésophiles où cette portion linéaire se limite généralement à un intervalle où l'inoculum varie de 1 à 4 (fig. 4). Cette réserve faite sur le plan théorique, les résultats expérimentaux obtenus nous ont conduit à utiliser une relation linéaire, particulièrement commode sur le plan pratique, d'autant qu'elle n'entraîne pas d'erreur d'interprétation systématique et grossière même si elle n'est pas exactement vérifiée.

Les résultats expérimentaux obtenus avec des suspensions de streptocoques lactiques mésophiles appartenant à des espèces différentes (fig. 4) nous ont par ailleurs conduit à comparer l'activité acidifiante de ces diverses suspensions à une même suspension de référence. Nous avons en effet estimé que, là encore, nous ne commettions pas d'erreur systématique et importante sur le plan expérimental. Dans le cas de Str. thermophilus, les deux souches étudiées, 302 et 21, nous ont donné des résultats légèrement différents. Dans le cas des lactobacilles thermophiles étudiés, les variations sont trop importantes d'une souche à une autre pour qu'il soit possible d'utiliser une suspension de référence unique. Il nous a paru au contraire nécessaire de définir pour chaque espèce, voire pour chaque souche, une suspension de référence. 


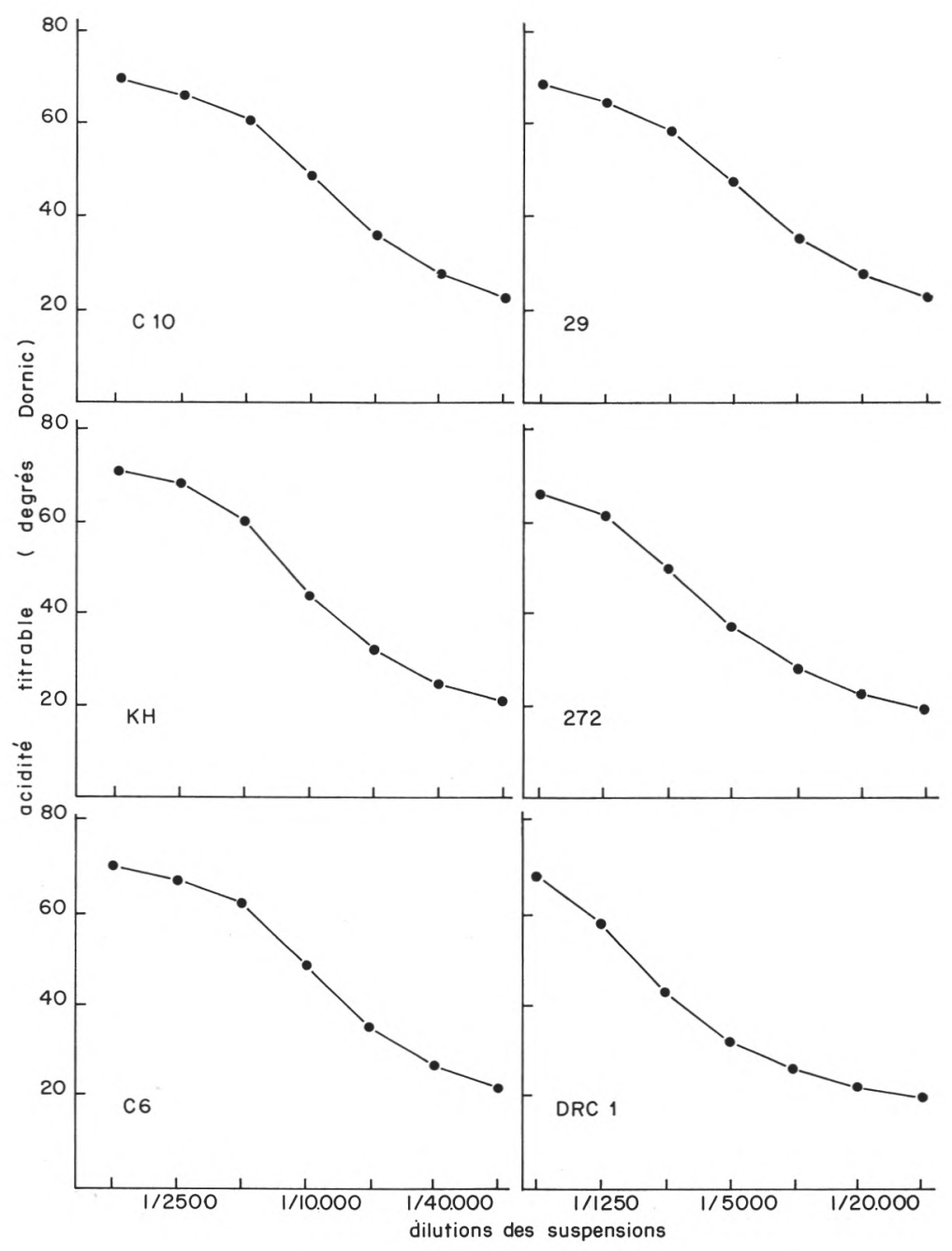

fig. 4

Variations de la quantité d'acide produite par différentes suspensions de streptocoques lactiques mésophiles, en fonction de l'inoculum.

Str. cremoris $\mathrm{KH}$, Str. diacetilactis DRC 1.

Str. lactis C 6, C 10, 29, 272. 
Cette méthode a été utilisée régulièrement depuis plusieurs années, dans l'étude de la préparation et de la conservation à l'état congelé des bactéries lactiques. Elle permet également d'estimer de manière satisfaisante les performances en fabrication des suspensions utilisées en technologie, en particulier dans le cas des streptocoques lactiques mésophiles. Il est toutefois nécessaire d'effectuer l'extrapolation des résultats obtenus avec prudence et réserves.

\section{Rés u m é}

Il a été montré expérimentalement qu'il existe, dans certaines limites, une relation linéaire entre la quantité d'acide produite, en culture sur lait, par une suspension de bactéries lactiques, et le logarithme de la quantité de bactéries initialement présentes dans l'inoculum. L'étude de suspensions différentes d'une même souche bactérienne a montré que les droites obtenues sont parallèles.

Ces données expérimentales ont permis de formuler une expression mathématique et de décrire une méthode de détermination graphique simple de l'activité acidifiante des suspensions de bactéries lactiques. Cette méthode postule qu'une suspension $A_{1}$ est deux fois (ou dix fois) plus active que la suspension $A_{2}$, si la suspension $A_{1}$ doit être diluée deux fois (ou dix fois) plus que $A_{2}$, pour obtenir la même quantité d'acide. Dans le cas des streptocoques lactiques mésophiles, il paraît possible d'utiliser un système de référence unique, pour toutes les souches. Par contre, dans le cas des lactobacilles thermophiles, et vraisemblablement de Str. thermophilus, il est nécessaire d'utiliser un système de référence particulier, spécifique de chaque souche ou de chaque espèce. La précision et la reproductibilité de la méthode varient suivant les organismes étudiés. Elles dépendent en particulier de la pente des droites obtenues expérimentalement. Par exemple, la quantité d'acide produite par deux dilutions successives au $1 / 2$ d'une même suspension varie entre $26^{\circ} \mathrm{D}$ dans le cas de $L$. helveticus 303 et 2,5० D dans le cas de Str. thermophilus 302.

Cette méthode a été utilisée dans l'étude de la préparation et de la conservation à l'état congelé des suspensions de bactéries lactiques.

\section{S u m m a ry}

\section{Estimation of the acid producing activity of concentrated frozen suspensions of lactic acid bacteria}

Experiments have showed a linear relation between the amount of lactic acid produced in milk by a suspension of lactic acid bacteria and the logarithm of the number of bacteria in the inoculum. The straight lines obtained with different suspensions of the same strain are parallel. 
From these data it is possible to express mathematically and to determine graphically the activity of a given suspension of lactic acid bacteria. The method is based on the assumption that a suspension $A_{1}$ is twice (or ten times) more active than a suspension $A_{2}$ of the same strain, if $A_{1}$ can be diluted twice (or ten times) more than $A_{2}$, to give the same acid production. In the case of mesophilic streptococci, it seems possible to use the same reference curve for all the strains. On the other hand, for thermophilic lactobacilli and probably also for Str. thermophilus, different reference curves, specific for each species or strain, must be used. The precision and the reproducibility of the method varie with the organisms used, and are dependant on the slope of the curves. For instance, the amount of lactic acid produced by two successive $1 / 2$ dilutions of the same suspension varies between $26^{\circ} \mathrm{D}(0,26 \mathrm{p} .100$ lactic acid $)$ in the case of $L$. helveticus 303 and 2,5 D (0,025 p. 100 lactic acid) in the case of Str. thermophilus 302.

The method has been used in a study on the preparation and the storage of frozen suspensions of lactic acid bacteria.

Reçu pour publication en septembre 1970.

\section{$R$ éfére nces}

[1] Accolas (J.P.) et Auclair (J.) (1967). - Conservation à l'état congelé de suspensions de bactéries lactiques concentrées sous faible volume. I. Bactéries lactiques mésophiles. Le Lait, 47, 253-260.

[2] Baumann (D. P.) (1964). - Preservation of lactic cultures. Ph. D. Thesis, Iowa State University of Science and Technology, Ames, Iowa.

[3] Baumann (D. P.) and Reinbold (G. W.) (1966). - Freezing of lactic cultures. J. Dairy Sci., 49, 259-264.

[4] Bergere (J. L.) (1968). - Production massive de cellules de streptocoques lactiques. I. Méthodes générales d'étude et facteurs de la croissance de Str. lactis C 10. Le Lait, 48, 1-11.

[5] Bergere (J. L.) et Hermier (J.) (1968), - La production massive de cellules de streptocoques lactiques. II. Croissance de Streptococcus lactis en milieu à $\mathrm{pH}$ constant. Le Lait, 48, 13-30.

[6] Bergere (J. L.) (1968). - Production massive de cellules de streptocoques lactiques. III. Production de différentes souches en culture à $\mathrm{pH}$ constant. Le Lait, 48, 131-139.

[7] Cowman (R. A.) and Speck (M. L.) (1965). - Ultra-low temperature storage of lactic streptococci. J. Dairy Sci., 48, 1531-1532.

[8] Foster (E. M.) (1962). - Symposium on lactic acid starter cultures. VI. Culture preservation. J. Dairy Sci., 45, 1290-1294.

[9] Gavin (M.) (1968). - La lyophilisation des cultures de yoghourt. Thèse $n^{\circ}$ 4227, Ecole Polytechnique Fédérale, Zürich.

[10] Lamprech (E. D.) and Foster (E. M.) (1963). - The survival of starter organisms in concentrated suspensions. J. appl. Bact., 26, 359-369.

[11] Lattuada (C. P.) (1964). - Survival of Streptococcus lactis after drying and storage. Ph. D. Thesis, The University of Wisconsin. 
[12] Peebles (M. M.) Gilliland (S. E.) and Speck (M. L.) (1969). - Preparation of concentrated lactic Streptococcus starters. Appl. Microbiol., 17, 805-810.

[13] Pont (E. G.) and Holloway (G. L.) (1968). - A new approach to the production of cheese starter. Aust. J. Dairy Technol., 23, 22-29.

[14] Reif (G. D.), Reinbold (G. W.), and Vedamuthu (E. R.) (1967). - Effect of frozen storage upon lactic cultures grown at 15 and 21 C. J. Dairy Sci., 50,945 ,

[15] Rousseaux (P.), Vassal (L.), Valles (E.), Auclair (J.) et Mocquot (G.) (1968). - Utilisation en fromagerie de Gruyère de suspensions concentrées et congelées de bactéries lactiques thermophiles. Le Lait, 48, 241-254.

[16] Stadhouders (J.), Jansen (L. A.) and Hup (G.) (1969). - Preservation of starters and mass production of starter bacteria. Neth. Milk Dairy J., 23, 182-199.

[17] Valles (E.) et Mocouot (G.) (1968). Préparation de suspensions concentrées et congelées de bactéries lactiques thermophiles destinées à la fromagerie. Le Lait, 48, 631-643.

[18] Vassal (L.) et Mocouot (G.) (1967). - Fermentation lactique accélérée en fromagerie grâce à l'emploi d'un nombre très élevé de bactéries. $L a$ Technique Laitière, $\mathrm{n}^{\circ}$ 541, 9-13.

\section{Remerciements}

Nous remercions vivement MM. Bergère et Hermier des suggestions et critiques qui nous ont été très utiles pour la rédaction du manuscrit.

\section{A n n e xe}

La relation linéaire existant entre la quantité d'acide produite y et le logarithme de la quantité de germes initialement présents dans l'inoculum (ou, plus généralement, entre y et le facteur de dilution $\mathrm{x}$ ), peut s'exprimer sous la forme générale $\mathrm{y}=\mathrm{k} \log _{2} \frac{\mathrm{x}}{\mathrm{x}_{0}}$, $\mathrm{k}$ et $\mathrm{x}_{0}$ étant des
constantes.

On en tire la forme exponentielle $\mathrm{x}=\mathrm{x}_{0} \frac{\mathrm{y}}{2 \mathrm{k}}$, qui permet de rapporter l'activité au facteur de dilution.

Considérons maintenant les droites obtenues avec deux suspensions différentes A et B d'une même souche bactérienne (fig. 5). Ces deux droites étant parallèles, il est possible de comparer les activités acidifiantes de ces deux suspensions, en exprimant le rapport $r=\frac{x_{1}}{x_{2}}$ des facteurs de dilution correspondant à une même production d'acide $\mathrm{y}_{1}$.

Exprimons la pente $\mathrm{k}$ de chacune des deux droites $\mathrm{A}$ et $\mathrm{B}$, en utilisant des valeurs connues $\mathrm{y}_{1}, \mathrm{y}_{2}$ et $\mathrm{y}_{3}$ correspondant aux facteurs de dilution $\mathrm{x}_{1}, \mathrm{x}_{2}$ et $\mathrm{x}_{3}$.

Dans le cas de la droite $\mathrm{B}, \mathrm{k}=\frac{\mathrm{y}_{1}-\mathrm{y}_{2}}{\log _{2} \frac{\mathrm{x}_{1}}{\mathrm{x}_{0}}-\log _{2} \frac{\mathrm{x}_{2}}{\mathrm{x}_{0}}}$ ou $\mathrm{k}=\frac{\mathrm{y}_{1}-\mathrm{y}_{2}}{\log _{2} \frac{\mathrm{x}_{1}}{\mathrm{x}_{2}}}(1)$.

De même, dans le cas de la droite $\mathrm{A}, \mathrm{X}=\frac{\mathrm{X}_{0}}{\mathrm{y}_{1}-\mathrm{y}_{3}}$ (2).

$$
\log _{2} \frac{\mathrm{X}_{2}}{\mathrm{X}_{3}}
$$


Si les dilutions ont été choisies de manière à ce que $\mathrm{x}_{2}=2 \mathrm{x}_{3}$, (2) s'écrit, dans ce cas, sous la forme simplifiée : $\mathrm{k}=\mathrm{y}_{1}-\mathrm{y}_{3}$ (3).

En combinant (1) et (3), on obtient alors l'équation suivante :

$$
\frac{\mathrm{y}_{1}-\mathrm{y}_{2}}{\mathrm{y}_{1}-\mathrm{y}_{3}}=\log _{2} \frac{\mathrm{x}_{1}}{\mathrm{x}_{2}}=\log _{2} \mathrm{r}
$$

qui permet d'exprimer le rapport $\frac{\mathrm{x}_{1}}{\mathrm{x}_{2}}$, sous les formes équivalentes :

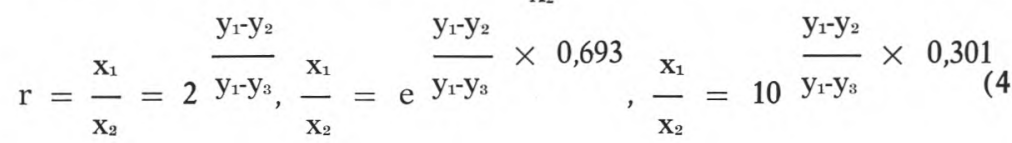

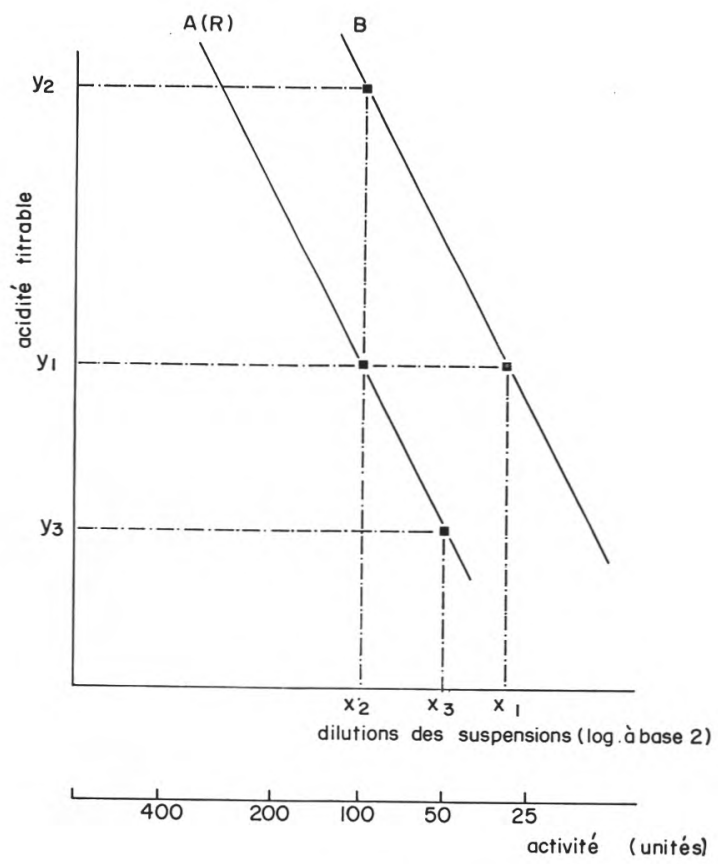

fig. 5

Supposons maintenant que la suspension A constitue la suspension de référence $R$. L'activité acidifiante de $R$ est par définition égale à 100 unités par $\mathrm{g}$. On peut alors écrire :

$$
\mathrm{r}=\frac{\text { activité de } \mathrm{A}}{\text { activité de } \mathrm{B}}=\frac{100}{\text { activité de } \mathrm{B}}
$$

Il est possible d'exprimer directement en unités l'activité de $\mathrm{B}$, à l'aide de la formule suivante : 
activité de $\mathrm{B}$ (unités) $=100 \times \frac{\mathrm{x}_{2}}{\mathrm{x}_{1}}$, en utilisant l'une quelconque des expressions (4) pour calculer $\frac{\mathrm{X}_{2}}{\mathrm{X}_{1}}$. On a par exemple :

$$
\frac{\mathrm{y}_{2}-\mathrm{y}_{1}}{\mathrm{y}_{1}-\mathrm{y}_{3}} \times 0,301
$$

activité de $\mathrm{B}$ (unités) $=100 \times 10$

Prenons un exemple. Dans le cas de L. helveticus 303, la suspension $\mathrm{R}$ est définie par les productions d'acide $\mathrm{y}_{1}=70^{\circ} \mathrm{D}$ à la dilution $1 / 1000$ et $\mathrm{y}_{3}=44^{\circ} \mathrm{D}$ à la dilution $1 / 2000$. Si une suspension inconnue $\mathrm{B}$ produit une acidité titrable $\mathrm{y}_{2}=82^{\circ} \mathrm{D}$ à la dilution $1 / 1000$, son activité exprimée en unités est égale à :

soit 138 unités.

$$
100 \times 10^{\frac{82-70}{70-44} \times 0,301}=100 \times 10^{0,139},
$$

Plus simplement, on peut effectuer cette détermination graphiquement, en utilisant un graphique en coordonnées semi-logarithmiques analogue à celui qui est représenté, à titre d'exemple pour L. helveticus 303, sur la figure 3. Dans ce cas, l'axe des abscisses est directement gradué en unités d'activité. La suspension de référence est définie arbitrairement par la droite R ; 100 unités d'activité correspondent, dans l'exemple choisi, à une suspension produisant une acidité titrable de $70^{\circ} \mathrm{D}$ à la dilution au $1 / 1$ 000. Pour chacune des deux suspensions inconnues, on mène du point correspondant à la quantité d'acide produite à la dilution au $1 / 1000$, une parallèle à l'axe des abscisses. Chacune de ces parallèles coupe la droite de référence $\mathrm{R}$ en un point auquel correspond en abscisse le nombre d'unités d'activité contenues par chacune des deux suspensions. 